EGU21-13203

https://doi.org/10.5194/egusphere-egu21-13203

EGU General Assembly 2021

(c) Author(s) 2021. This work is distributed under

the Creative Commons Attribution 4.0 License.

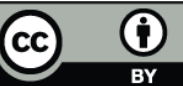

\title{
Modular designed Apps - an opportunity to standardize data collection methods and to encourage the reuse of software
}

\author{
Sina C. Truckenbrodt ${ }^{1,2}$, Maximilian Enderling ${ }^{2}$, Carsten Pathe ${ }^{1,2}$, Erik Borg ${ }^{3,4}$, Christiane C. \\ Schmullius ${ }^{1}$, and Friederike Klan ${ }^{2}$ \\ ${ }^{1}$ Friedrich-Schiller-Universität Jena, Institute of Geography, Earth Observation, Jena, Germany (sina.truckenbrodt@uni- \\ jena.de) \\ ${ }^{2}$ German Aerospace Center (DLR) Jena, Institute of Data Science, Citizen Science, Jena, Germany \\ ${ }^{3}$ German Aerospace Center (DLR) Neustrelitz, German Remote Sensing Data Center, National Ground Segment, Neustrelitz, \\ Germany \\ ${ }^{4}$ University of Applied Sciences Neubrandenburg, Geodesy and Geoinformatics, Neubrandenburg, Germany
}

Data collection strategies vary among different citizen science projects. This complicates the intercomparability of parameter values acquired in different studies (e.g., methodological and scale issues) and results in variable data quality. This creates problems regarding the merging of different data sets and hampers the reuse of data from different projects. Modular designed applications for mobile devices (Apps) represent a framework that helps to foster the standardisation of data collection methods. While they encourage the reuse of the software, they provide enough flexibility for an adjustment in accordance with the research question(s) of interest.

The currently developed App "FieldMApp" offers such a framework running under Android and iOS. The related concept includes predefined frame functionalities, like settings for the user account and the user interface, and adaptable application-related functionalities. The latter comprise several modules that are categorized as sensor test, basic functionality, parameter collection and data quality collection modules. The interdependencies of these modules are documented in a wiki. This enables an individual and context-based selection of functionalities. The FieldMApp is based on open-source software libraries (Xamarin, Open Development Kit (ODK), SQLite, CoreCLR-NCalc, LusoV.YamarinUsbSerialForAndroid, Newtonsoft.Json, SharpZipLib) and will be published as open-source software. Hence, the existing catalogue of functionalities can be augmented in the future. The premise for such extensions is that modules are published together with smart, universally applicable data quality recording routines and a proper documentation in the wiki.

In this contribution, we present the concept and the structure of the FieldMApp and some current fields of application that are related to the cultivation of arable land, soil mapping, forest monitoring, and Earth Observation. The extension of the functionality catalogue is exemplified by the newly implemented speech recognition module. A related quality recording routine will be introduced. With this contribution we would like to encourage citizens and scientists to elicit which 
requirements such an App should fulfil from their point of view. 